\title{
Quantitative Analysis of Myocardial Perfusion SPECT Anatomically Guided by Coregistered 64-Slice Coronary CT Angiography
}

Piotr J. Slomka ${ }^{1,2}$, Victor Y. Cheng ${ }^{1}$, Damini Dey ${ }^{1,2}$, Jonghye Woo ${ }^{1}$, Amit Ramesh ${ }^{1}$, Serge Van Kriekinge ${ }^{1,2}$, Yasuzuki Suzuki ${ }^{1}$, Yaron Elad ${ }^{3}$, Ronald Karlsberg ${ }^{3}$, Daniel S. Berman ${ }^{1,2}$, and Guido Germano ${ }^{1,2}$

${ }^{1}$ Departments of Imaging and Medicine, Cedars-Sinai Medical Center, Los Angeles, California; ${ }^{2}$ Department of Medicine, David Geffen School of Medicine at UCLA, Los Angeles, California; and ${ }^{3}$ Cardiovascular Medical Group, Beverly Hills, California

Sequential testing by coronary CT angiography (CTA) and myocardial perfusion SPECT (MPS) obtained on stand-alone scanners may be needed to diagnose coronary artery disease in equivocal cases. We have developed an automated technique for MPS-CTA registration and demonstrate its utility for improved MPS quantification by guiding the coregistered physiologic (MPS) with anatomic CTA information. Methods: Automated registration of MPS left ventricular (LV) surfaces with CTA coronary trees was accomplished by iterative minimization of voxel differences between presegmented CTA volumes and motion-frozen MPS data. Studies of 35 sequential patients ( 26 men; mean age, $67 \pm 12$ y) with 64-slice coronary CTA, MPS, and available results of the invasive coronary angiography performed within 3 mo were retrospectively analyzed. Three-dimensional coronary vessels and CTA slices were extracted and fused with quantitative MPS results mapped on LV surfaces and MPS coronary regions. Automatically coregistered CTA images and extracted trees were used to correct the MPS contours and to adjust the standard vascular region definitions for MPS quantification. Results: Automated coregistration of MPS and coronary CTA had the success rate of $96 \%$ as assessed visually; the average errors were $4.3 \pm 3.3 \mathrm{~mm}$ in translation and $1.5 \pm 2.6$ degrees in rotation on stress and $4.2 \pm 3.1 \mathrm{~mm}$ in translation and $1.7 \pm 3.2$ degrees in rotation on rest. MPS vascular region definition was adjusted in 17 studies, and LV contours were adjusted in 11 studies using coregistered CTA images as a guide. CTA-guided myocardial perfusion analysis, compared with standard MPS analysis, resulted in improved area under the receiver-operating-characteristic $(\mathrm{ROC})$ curves for the detection of right coronary artery (RCA) and left circumflex artery (LCX) lesions ( $0.84 \pm 0.08$ vs. $0.70 \pm 0.11$ for $L C X, P=0.03$, and $0.92 \pm 0.05$ vs. $0.75 \pm 0.09$ for RCA, $P=0.02)$. Conclusion: Software image coregistration of stand-alone coronary CTA and MPS obtained on separate scanners can be performed rapidly and automatically, allowing CTA-guided contour and vascular territory adjustment on MPS for improved quantitative MPS analysis.

Received Mar. 6, 2009; revision accepted Jun. 23, 2009.

For correspondence or reprints contact: Piotr Slomka, Cedars-Sinai Medical Center, Department of Imaging, \#A047 8700 Beverly Blvd., Los Angeles, CA 90048.

E-mail: Piotr.Slomka@cshs.org

COPYRIGHT @ 2009 by the Society of Nuclear Medicine, Inc.
Key Words: myocardial perfusion imaging; CT angiography; image registration; image fusion; coronary artery disease; image quantification; SPECT

J Nucl Med 2009; 50:1621-1630

DOI: 10.2967/jnumed.109.063982

$\mathbf{R}$ ecent advances in cardiac coronary CT angiography (CTA) have allowed the precise localization and classification of coronary artery plaques (1) and the depiction of coronary anatomy. At the same time, myocardial perfusion SPECT (MPS) continues to be a mainstream imaging modality for the detection and estimation of the severity of ischemia caused by coronary lesions. Although either CTA or MPS will frequently produce adequate diagnostic information for the referring physician, a subset of patients undergoes sequential testing by both modalities because of inconclusive results obtained by 1 of the tests $(2,3)$. In such cases, imaging results are often challenging to interpret because of the presence of artifacts or equivocal findings in at least 1 modality.

It has been suggested that the visual analysis of fused MPS and coronary CTA images can synergistically improve the diagnostic value of sequential combined imaging (4), and manual tools for the purpose of combined visual analysis have been developed (5). However, the need for the interactive alignment complicates clinical protocols, introduces manual steps, and reduces practical usability of such tools. Furthermore, current coronary CTA and MPS image fusion tools were proposed solely to improve subjective visual analysis and do not take advantage of the available CTA anatomic information, which can be used to refine quantitative MPS results.

In this work, we aimed to develop a novel and practical tool for rapid automatic coregistration, visualization, and combined quantification of coronary CTA and MPS obtained from stand-alone scanners in different scanning sessions. Furthermore, we showed that coregistered MPS-CTA data can be used to improve quantitative MPS analysis. 


\section{MATERIALS AND METHODS}

\section{Patient Selection}

Between October 2005 and May 2007, we identified retrospectively 40 consecutive patients who underwent myocardial MPS, CTA, and invasive coronary angiography (ICA) within a 90-d period at Cedars-Sinai Medical Center (site A) or a neighboring outpatient imaging center (Cardiovascular Medical Group) (site B). The number of scans in which both CTA and MPS were performed was estimated at $5 \%-10 \%$ of the MPS volume at both sites, and requiring ICA results further reduced the eligible population in this study. For 2 patients, the relevant imaging data could not be retrieved from the image archive, and these cases were excluded. A total of 22 patients were imaged for the evaluation of symptoms (either chest pain or dyspnea; 8 had prior myocardial infarction). The remaining 16 patients were asymptomatic. The imaging indications were for post-myocardial infarction ( 3 cases) or post-percutaneous coronary intervention (3 cases) risk stratification and for risk stratification without prior event (10 cases). Furthermore, 3 patients were excluded from further analysis because of coronary artery bypass graft surgery, which would present difficulty in the assessment of whether ischemia would be expected in the distribution of bypassed vessels in which regions subtended by proximal branch vessels may be ischemic despite open grafts to the distal vessel. The remaining 35 patients ( 26 men, 9 women; mean age, $67 \pm 12$ y) were included in our analysis. In 5 cases, CTA and MPS were performed on the same day; in 20 cases, CTA was performed after MPS (range, 1-49 d; median, $9 \mathrm{~d}$ ); and in 10 cases, MPS was performed after CTA (range, 1-73 d; median, $13 \mathrm{~d}$ ). Table 1 contains patient characteristics. This study was approved by the Institutional Review Boards at CedarsSinai Medical Center and Cardiovascular Medical Group.

\section{CT Image Acquisition}

Because the presence of coronary calcium can significantly affect CTA image quality, CT unenhanced scans were obtained, and Agatston CT calcium scores were computed on a ScImage workstation $(6,7)$ (site A) or with SmartScore software (Advantage Workstation; GE Healthcare) (site B). Coronary CTA was performed on the SOMATOM Definition dual-source CT (DSCT) scanner (Siemens Medical Systems) (site A) or LightSpeed VCT 64-slice CT scanner (GE Healthcare) (site B). A prescan $\beta$-blockade, with a target heart rate less than 60 beats/min for VCT and less than 70 beats/min for DSCT, was performed, and an unenhanced coronary calcium scan was obtained. Electrocardiogram (ECG)-gated helical CTA was performed during a 9- to 12-s breath hold by powerinjecting $90-100 \mathrm{~mL}$ of intravenous contrast. The following were scanning parameters: heart rate-dependent pitch; $0.33 \mathrm{~s}$ (DSCT) to

\begin{tabular}{lr}
\hline TABLE 1. Patient Characteristics \\
\hline Characteristic & Mean value \\
Sex, male & $26(74 \%)$ \\
Age $(\mathrm{y})$ & $67 \pm 12$ \\
Asymptomatic & $15(43 \%)$ \\
Myocardial infarction & $11(31 \%)$ \\
Weight (kg) & $80 \pm 16$ \\
Body mass index & $27.6 \pm 5.7$ \\
& \\
\hline & \\
Data are mean \pm SD, or number followed by percentage in \\
parenthes $(n=35)$.
\end{tabular}

$0.35 \mathrm{~s}$ (VCT) gantry rotation time; detector collimation, $0.625 \mathrm{~mm}$; tube voltage, $120 \mathrm{kVp}$; and tube current, 300-700 mAs. ECG-based dose modulation $40 \%-80 \%$ of the cardiac cycle was used to limit radiation dose.

\section{Coronary CTA Image Reconstruction}

Retrospectively gated reconstruction of raw CTA data was performed at $40 \%-80 \%$ of the R-R interval using the following parameters: 0.6 - to $0.75-\mathrm{mm}$ slice thickness, $250-\mathrm{mm}$ field of view, and $512 \times 512$ matrix. Coronary arterial trees were extracted from the best-quality phase using vendors' software and transferred to a Windows workstation for MPS-CTA fusion.

\section{CTA Image Evaluation}

Coronary visual analysis was performed with Circulation software packages (Siemens Medical Systems) and an Advantage Workstation (version 7.1; GE Healthcare). An experienced clinical coronary CTA reader (with $>300$ previous coronary CTA interpretations), unaware of MPS and ICA results, assessed all coronary segments greater than or equal to $1.5 \mathrm{~mm}$ in diameter by evaluating standard axial images, oblique multiplanar reformations, oblique maximum-intensity projections, or centerline-based curved multiplanar reformations (8). Each segment was visually evaluated for the presence and degree of stenosis. Any stenosis narrowing the luminal diameter by at least $50 \%$ or at least $70 \%$ was recorded. If a segment could not be assessed because of artifacts, no stenosis was recorded.

\section{ICA Image Acquisition and Evaluation}

ICA was performed with a standard catheterization technique. All ICA acquisitions used the same iodinated contrast (Visipaque; GE Healthcare). After ICA, a clinical interventional cardiologist, unaware of coronary CTA and MPS results, evaluated acquired images on an offline display station. For each coronary segment greater than or equal to $1.5 \mathrm{~mm}$ in diameter, this cardiologist determined by visual inspection whether luminal diameter narrowing $\geq 50 \%$ or $\geq 70 \%$ was present. For the purposes of comparison with MPS and CTA data, left main stenosis $\geq 50 \%$ was considered as significant for the left anterior descending artery and left circumflex artery (LCX) territories; the ramus intermedius vessel, if present, was assigned to the LCX territory. Angiographic findings are presented in Table 2.

\begin{tabular}{|cr|}
\hline TABLE 2. Angiographic Characteristics of Data $(n=35)$ \\
\hline Characteristic & Value \\
$\geq 70 \%$ stenosis & $27(77 \%)$ \\
$\geq 50 \%$ stenosis & $32(91 \%)$ \\
Left main $(\geq 50 \%)$ & $6(17 \%)$ \\
LAD $(\geq 70 \%)$ & $21(60 \%)$ \\
LCX $(\geq 70 \%)$ & $12(34 \%)$ \\
RCA $(\geq 70 \%)$ & $15(43 \%)$ \\
No stenosis $\geq 50 \%$ & $3(9 \%)$ \\
No stenosis $\geq 70 \%$ & $8(23 \%)$ \\
1-vessel disease $(\geq 70 \%)$ & $11(31 \%)$ \\
2-vessel disease $(\geq 70 \%)$ & $11(31 \%)$ \\
3-vessel disease $(\geq 70 \%)$ & $5(14 \%)$ \\
& \\
\hline &
\end{tabular}




\section{MPS Protocol}

Studies were performed at the 2 participating sites with the same dual-isotope (thallium-technetium) protocol previously described (9). MPS acquisitions were performed with noncircular orbits, obtaining 64 projections over $180^{\circ}\left(45^{\circ}\right.$ right anterior oblique to $45^{\circ}$ left posterior oblique). Images were acquired on Philips CardioMD, Philips Forte, and Siemens e.cam cameras in 1- or 2-d protocols. A stress scan was obtained with exercise, an adenosine injection, or an adenosine-walk protocol (10). All images were subject to standard clinical quality control measures, and all were reconstructed at site B. No attenuation or scatter correction was used. Gated images were reconstructed with filtered backprojection and a Butterworth filter (cutoff, 0.83 cycles/cm; order, 5) to the original transverse orientation. Patients from site A had 16-frame gated MPS, and patients from site B had 8-frame gated MPS.

\section{Image Processing}

An overview of image processing is presented in Figure 1.

Motion-Frozen Processing. Gated images were processed with a motion-frozen algorithm as previously described (11) to provide static MPS images in end-diastolic (ED) phase to match the diastolic cardiac phase of coronary CTA. Briefly, in the motion-frozen technique, derived left ventricular (LV) contours (12-14) are used in combination with a thin-plate spline image warping $(15,16)$.

Automated Registration. The primary difficulty in accurate registration of MPS with coronary CTA is that different anatomic features appear on these images. Therefore, standard automated registration procedures (17) are prone to large errors. In particular, there could be severe perfusion defects present on MPS images that are not visualized in the myocardium on the CTA scan, which can present difficulties for generic registration algorithms. To provide a robust registration approach, we registered presegmented MPS volumes using the LV segmentation algorithm of quantitative gated SPECT (14) and the additional segmentation of the blood-pool region, derived from the MPS LV definition. In addition, to match cardiac phases between MPS and coronary CTA, we aligned coronary CTA-reconstructed slices in diastolic phases with segmented motion-frozen slices of SPECT created from gated images. For both CTA and MPS, transverse image orientation was used during the registration process.

The MPS-CTA registration algorithm was implemented as follows. We initially aligned the center of mass of the segmented left ventricle on MPS with the geometric center of the reconstructed coronary CTA image domain. Subsequently, we performed iterative registration between CTA and segmented (LV, blood-pool) MPS images to find the transformation $T$ that maps the source volume $J$ (CTA) into correspondence with target volume $I$ (MPS). We considered 6 rigid-body parameters for the MPS-CTA registration, assuming the cardiac phases are matched. $S_{I}$ was the segmented MPS volume, which consisted of blood-pool, wall, and background regions. We used the sum of squared differences as a similarity measure and replaced the original MPS intensities with preassigned values for the blood pool, myocardium, and surrounding structures, which are derived from average Hounsfield unit values recorded by CTA in these regions. The proposed registration cost function $E$ is given by

$$
E(x, T(x))=\int_{\Omega}\left(S_{I}(x+T(x))-J * G_{\sigma}(x)\right)^{2} d x
$$

where * denotes the convolution operator, $G_{\sigma}$ is gaussian kernel with $\mathrm{SD} \sigma$, and $\Omega$ is the image volume. We applied gaussian filtering (with 3-mm kernel) to the CTA volume to suppress noise and match image resolution to MPS. To minimize the cost function, we used iterative gradient descent and used a coarseto-fine multiresolution scheme ( 3 levels) that helped to avoid local minima while achieving computational efficiency and robustness. We used the Insight Toolkit registration libraries (18) for the implementation of the iterative registration. All algorithms were implemented in $\mathrm{C}++$.

Validation of Automatic Registration. The automatic registration algorithm was assessed qualitatively and quantitatively for all 35 cases (total of 70 stress and rest datasets). Quantitative error analysis was performed by comparison to the expert manual alignment. The manual alignment parameters (3 translations and 3 rotations) were recorded for the comparison with the automated registration. Visual alignment was performed without knowledge of the automatic results.

Vessel Extraction and 3-Dimensional (3D) Visualization. For the $3 \mathrm{D}$ visualization of coronary vessels superimposed with MPS surfaces, we used the processing information obtained from coronary CTA analysis on the vendors' workstation. Subsequently, volume rendering was performed in OpenGL with 2-dimensional/ 3D textures and preassigned color tables with varying red-greenblue intensities and opacities to maximize the contrast opacity, provide realistic display (blood/tissue, pink/red; calcium, white), and minimize the influence of neighboring tissue such as fat (19).

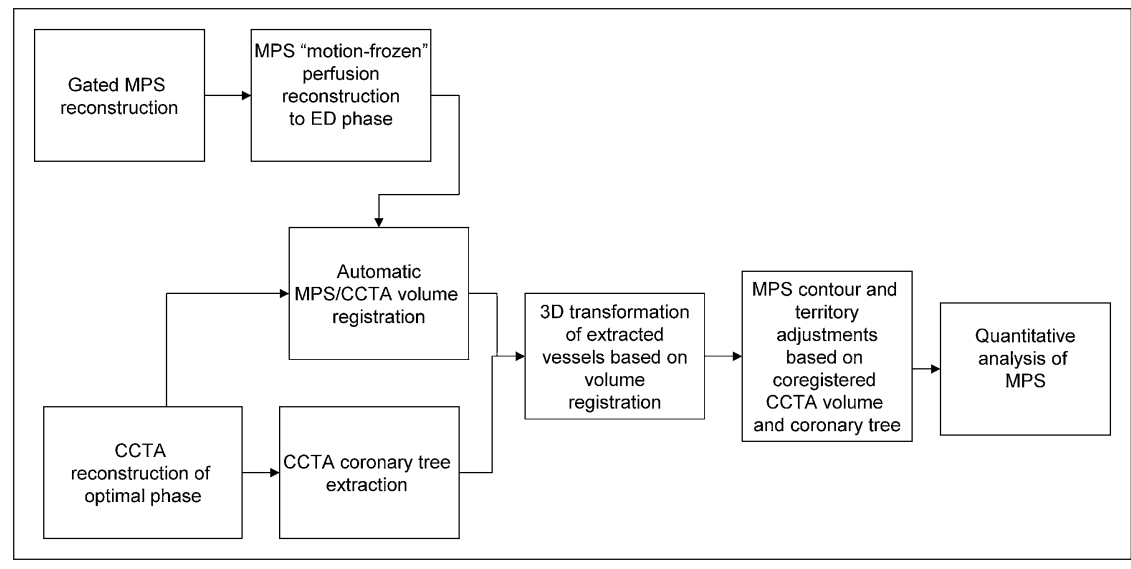

FIGURE 1. Overview of image processing. CCTA = coronary CTA. 
Segmented CTA voxel maps could then be directly rendered in 3 dimensions with OpenGL methods within quantitative perfusion SPECT (QPS) and within the same coordinates as the epicardial 3D surface display with overlaid MPS function and perfusion information. We used a level-of-detail rendering technique: a high-resolution image for final static display and a lower resolution during the user interaction and manipulation. In both cases, a 3D bounding box was used to speed up the volume rendering of coronary arteries. The bounding box was directly computed from the coronary mask or from the coronary tree coordinates. The rendering was integrated with the standard 3D MPS epicardial surfaces. Raw perfusion information of quantitative blackout maps could be displayed on the epicardial surface. Stress and rest images had the same coronary CTA image superimposed. These segmented coronary trees derived from CTA were used only for display purposes and were not used in any way during the image registration process. The image registration algorithm was based on only the original transverse datasets.

CTA-Guided MPS Contour and Territory Adjustment. Fused coronary CTA and MPS images were evaluated with overlaid contours in multiplanar orientations (both cardiac axis and orthogonal views) in the fusion page of the QPS program. If discrepancies between the MPS valve plane position and the location on CTA could be seen on the fused images, the contour was manually adjusted in the standard Manual QPS page with Mask and Constrain options; subsequently, a new contour was verified by the CTA fusion. Next, the default vascular territory boundaries were overlaid with the 3D LV MPS surfaces, with color-coded perfusion information and with a coregistered volume-rendered segmented 3D coronary tree as described above. Vascular territories could then be adjusted segment by segment (based on a 17-segment American Heart Association model), using anatomic information provided by the coronary CTA. If MPS contours or vascular territories needed to be adjusted on the basis of the comparison with CTA, the quantitative MPS analysis was repeated with modified contours and territories.

MPS perfusion analysis was performed individually for each vessel with the Group function in QPS, in which 17 segments are assigned to a vascular territory on the basis of the perfusion-defect pattern $(20,21)$. The portion of the total perfusion deficit (TPD) corresponding to a given territory was used for the automated quantification in each vessel, with the threshold of $2 \%$ as previously established (21). The MPS quantification results were recorded before and after adjustments based on the fused coronary data.

\section{Statistical Analysis}

All continuous variables are expressed as mean \pm SD. Paired $t$ tests were used to compare differences in paired continuous data, and McNemar tests were used to compare differences in paired discrete data. For unpaired continuous data, 1-way ANOVA was used. All statistical tests were 2-tailed, and a $P$ value of less than 0.05 was considered significant. Receiver-operating-characteristic (ROC) curves were constructed and compared using the AnalyzeIt 2.09 software, which implemented the Delong-Delong method for ROC comparisons (22).

\section{RESULTS}

Of 35 cases with all 3 scans (CTA, MPS, and ICA) available, 20 patients underwent CTA after MPS and 15 underwent MPS after CTA. In cases in which CTA was performed after MPS, 11 had equivocal reversible defects on visual evaluation of MPS (interpreted as probably normal, borderline, or probably abnormal), and 9 were referred for CTA because the caretaking physician believed defects seen on MPS were discordant with the patient's clinical status or suspected multivessel disease. In cases in which patients underwent MPS after CTA, 7 had at least 1 nondiagnostic major coronary segment on CTA, and 4 had maximal luminal stenosis in the LAD estimated at $50 \%$ and considered of borderline significance. Additionally, 4 patients were referred for MPS after CTA because the physician requested an assessment of hypoperfusion.

\section{Baseline Findings}

In the 35 cases studied, the unenhanced CT calcium score was available in 33 cases. In these cases, the average was $942 \pm 1,530$ (range, 0-7,781). Heavy calcification (score $>500$ ) was present in 15 of 33 datasets. In addition, 10 cases demonstrated motion artifacts on CTA. Interpretation difficulties were noted in 9 cases. The presence of significant CT disease was noted in 27 of 35 studies, with 6 LCX lesions, 11 right coronary artery (RCA) lesions, 21 LAD lesions, and 2 left main lesions.

MPS ejection fractions were $57.4 \% \pm 14 \%$ (range, $32 \%-83 \%$ ) on stress and $57.2 \% \pm 14$ (range, $25 \%-83 \%$ ) on rest. Transischemic dilation was $1.15 \pm 0.14$ (range, 0.96-1.4). Visually, MPS findings were assessed as normal in 3 cases, as probably normal in 3 cases, borderline in 6 cases, probably abnormal in 1 case, and abnormal in 22 cases. Quantitatively, TPD was $16.5 \% \pm 12.7 \%$ on stress (range, $0 \%-44 \%$ ) and $5.6 \% \pm 8.1 \%$ on rest (range, $0 \%-25 \%$ ).

\section{Registration Algorithm}

The execution speed of automated registration was approximately $1-2 \mathrm{~s}$ per study on a $3.0-\mathrm{GHz}$ Pentium (Intel) dual-core computer. The automatic volume registration of motion-frozen MPS with CTA was successful in 33 of 35 stress and 34 of 35 rest studies as assessed qualitatively, with an overall success rate of $96 \%$. In 1 patient, because of the unusually high blood-pool contrast intensity on coronary CTA, registration failed for both stress and rest studies; the error occurred because of inadequate matching of assigned blood-pool contrast with the actual CT value in the blood-pool region. All 3 failed cases were women with small hearts (motion-frozen stress diastolic volumes, 29-52 $\mathrm{mL}$ on MPS). These results were easily corrected by interactive alignment. The quantitative registration errors obtained by comparison with the expert observer alignment are shown in Table 3. There were no significant differences between errors in different directions or between studies from 2 different systems. The errors ranged from 0 to $10 \mathrm{~mm}$. Figure 2 shows an example of the fused images obtained by the automated coregistration of MPS with CTA. 


\begin{tabular}{|c|c|c|c|c|c|c|}
\hline \multirow[b]{2}{*}{ Study } & \multicolumn{3}{|c|}{ Translations (mm) } & \multicolumn{3}{|c|}{ Rotations (degrees) } \\
\hline & $x$ & $y$ & $z$ & $x y$ & $x z$ & $y z$ \\
\hline Rest & $4.1 \pm 3.5$ & $4.0 \pm 3.6$ & $3.0 \pm 2.5$ & $2.4 \pm 3.5$ & $1.4 \pm 3.3$ & $1.3 \pm 2.6$ \\
\hline Stress & $4.4 \pm 3.3$ & $4.4 \pm 3.4$ & $3.4 \pm 2.4$ & $2.3 \pm 3.3$ & $1.2 \pm 3.0$ & $1.7 \pm 3.2$ \\
\hline
\end{tabular}

\section{Contour and Territory Adjustments}

MPS vascular region definitions were adjusted in 17 studies, and LV contours (valve plane location) were adjusted in 11 studies using coregistered coronary CTA images as a guide. The territory adjustment modified perfusion results for a specific vessel but not the overall perfusion deficit per study. The MPS contour adjustment modified overall TPD perfusion results in 7 of $35(20 \%)$ of the cases by more than $2 \%$. The territory adjustments modified perfusion in a specific vessel but did not change the global perfusion measure per study.

\section{Combined Performance for Coronary Artery Disease (CAD) Detection}

The areas under the ROC curve for the detection of disease in specific vessels are shown in Table 4, and corresponding ROC curves are shown in Figure 3. Sensitivities and specificities for the detection of disease in LAD, LCX, and LAD arteries were $67 \%$ and $50 \%, 67 \%$ and $83 \%$, and $67 \%$ and $60 \%$, respectively, for MPS and $76 \%$ and $71 \%, 75 \%$ and $100 \%$, and $87 \%$ and $85 \%$, respectively, for CTA-guided MPS ( $P=0.025$ for RCA). CTA-guided MPS analysis correctly identified 17 of 21 LAD, 9 of 12 LCX, and 13 of 15 RCA lesions corresponding to $\geq 70 \%$ stenosis on invasive angiography, compared with 17 of 21,6 of 12 , and 10 of 15, respectively, by CTA alone and with 14 of 21, 8 of 12 , and 10 of 15 , respectively, by MPS quantification unaided by CTA. If a simple either/or (CTA or CTA-guided MPS) positive criterion was applied for the detection of significant disease, the number of detected lesions increased to 19 of 21 for LAD, to 10 of 12 for LCX, and to 13 to 15 for RCA. Anatomic CTA-guided MPS reduced the number of discordant cases between CTA and quantitative MPS from 12 to 9 for LAD, from 8 to 5 for LCX, and from 12 to 6 for RCA. CTA-guided MPS agreed with angiography in 4 of 9 discordant cases for LAD, 4 of 5 cases for LCX, and 3 of 6 for RCA.

In Figure 4, we show an example of MPS contour adjustment (valve plane position) based on MPS fusion. In Figure 5, we demonstrate an example of contour adjustment and territory adjustment, which was performed after visual inspection of coregistered MPS-CTA images and quantitative MPS results obtained before and after these adjustments.
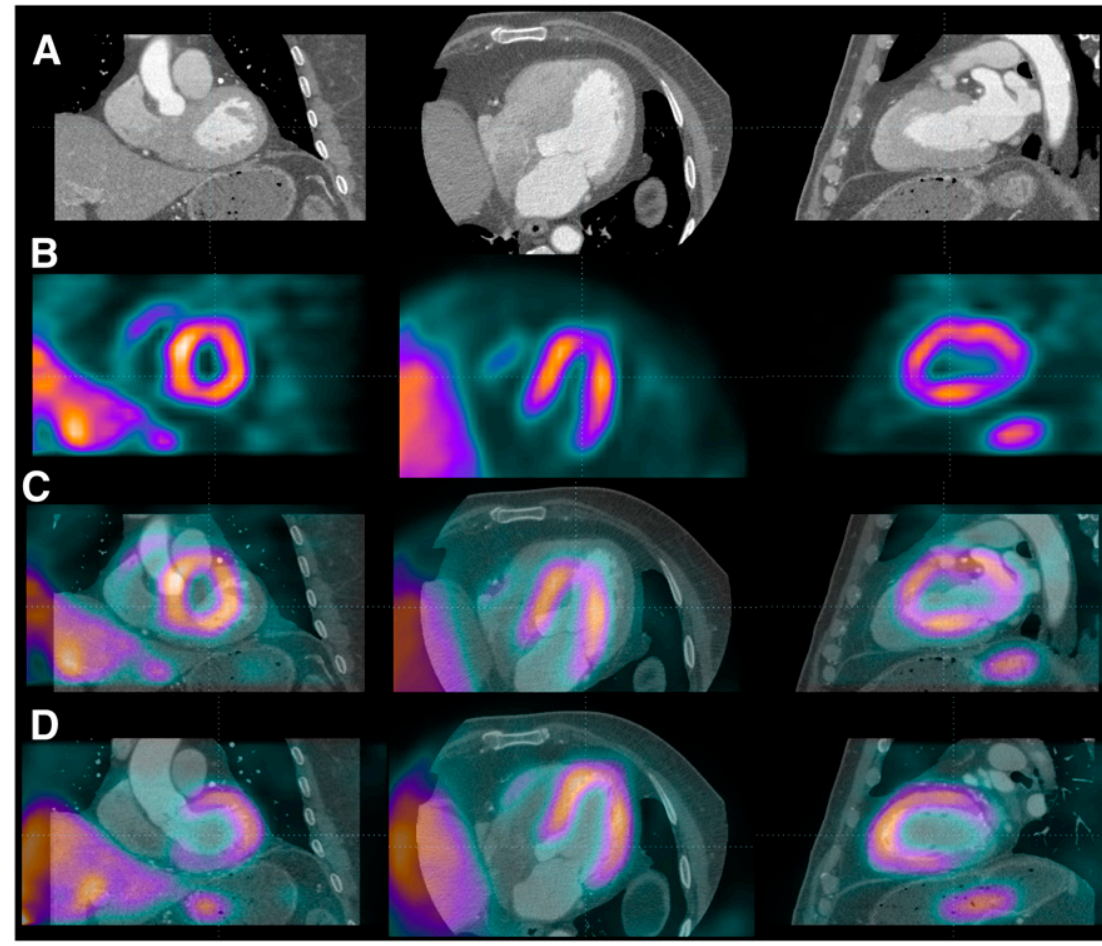
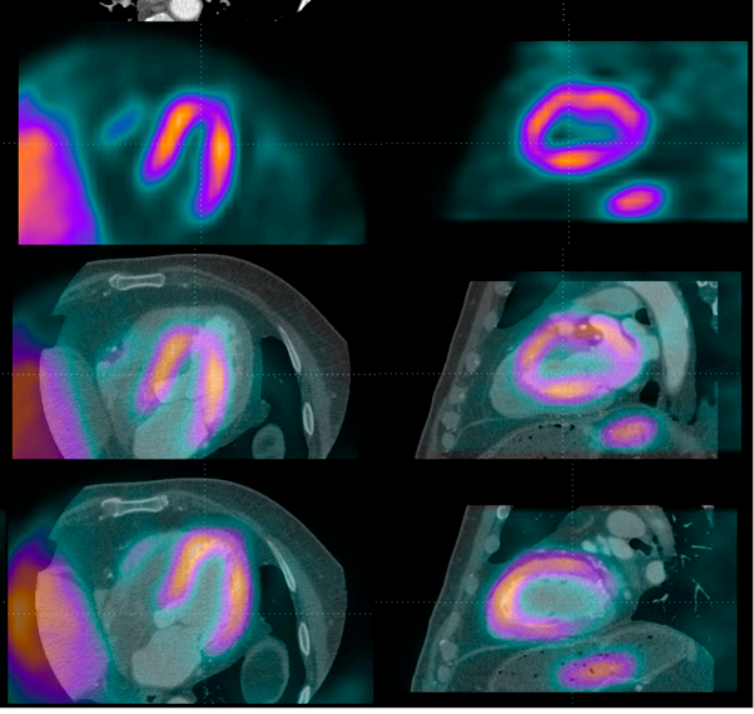

FIGURE 2. Automated volume alignment of CTA and MPS. Rows from top to bottom show in multiplanar orientations original CTA images (A), original MPS images (B), fused unregistered MPS and CTA images (C), and same images after automated volume registration (D). Subsequently, 3D transformation parameters obtained from volume registration can be used to align associated coronary trees with MPS surfaces. 
TABLE 4. Areas Under ROC Curves for Detection of CAD ( $\geq 70 \%$ Luminal Stenosis) in Individual Vessels

$\begin{array}{lccc}\begin{array}{c}\text { Areas under } \\ \quad \text { ROC curve }\end{array} & \text { LAD } & \text { LCX } & \text { RCA } \\ \text { MPS } & 0.74 \pm 0.08 & 0.70 \pm 0.11 & 0.75 \pm 0.09 \\ \text { CTA } & 0.76 \pm 0.09 & 0.75 \pm 0.09 & 0.81 \pm 0.07 \\ \begin{array}{c}\text { CTA-guided } \\ \quad \text { MPS }\end{array} & 0.78 \pm 0.08 & 0.84 \pm 0.08^{*} & 0.92 \pm 0.05^{*}\end{array}$

*Significantly different from MPS.

\section{DISCUSSION}

Software image fusion of coronary CTA and MPS has been proposed before to improve visual analysis of combined data from separate $(4,5)$ or hybrid scanners $(23)$. Our work introduces several novel technical concepts related to this application, namely fully automatic registration, the improvement of MPS quantification based on coregistered CTA, and the use of motion-frozen MPS data. We demonstrated that accurate and robust automatic image registration of motion-frozen MPS and coronary CTA can be performed for data obtained on stand-alone scanners in as short as 1-2 s, with a success rate of $96 \%$. Previous studies of MPScoronary CTA fusion required manual alignment of the image data $(4,5)$; it is likely that the automatic registration will allow MPS-CTA cardiac fusion in clinical practice whenever both datasets are available. We demonstrated that MPS contours-specifically the mitral valve plane position-can be adjusted on the basis of the CTA anatomic volume data. Furthermore, we showed that the computerized MPS vascular region definitions can be modified on the basis of coregistered coronary CTA anatomy; subsequently, the quantitative results can be reassigned to the correct territories, resulting in improved diagnostic performance, especially for LCX and RCA lesions.

A previous report of the clinical application of CTAMPS image fusion, compared with side-by-side analysis of stand-alone CTA and MPS, demonstrated that the correlation of stenosed segments with perfusion lesions allowed the significant reduction of equivocal findings (4). In our work, we show that CT-guided adjustment of contours and territories on MPS after image coregistration increases the diagnostic performance (area under the ROC curves) for the detection of coronary disease. Overall accuracy may be improved further by a combined visual analysis scheme that takes into account the relative size and the severity of the stenosis and the presence of artifacts on either of the 2 scans in a given location. When stand-alone CTA or MPS is insufficient to diagnose or localize CAD, CTA-guided MPS quantification can have an important role in increasing overall accuracy by lowering the number of discrepant findings. Because we studied a biased population with a high prevalence of equivocal results on the initial imaging test, we expect that most of the general MPS population will not significantly benefit from CTA-mediated contour and territory adjustments of MPS.

Although the automatic coregistration designed for coronary CTA and MPS volumes and CTA-guided MPS analysis have not been previously proposed, some related work has been reported. Faber et al. (24) developed a method of registration of 3D coronary anatomy reconstructed from invasive coronary angiography with MPS surfaces (24). They used a surface-to-point matching technique (25), which was modified to use coronary vessel information. Conceivably, such a registration approach could also be applied to the coronary CTA data; however, several of the arteries (RCA, left main) can be positioned some distance away from the myocardium, compromising the robustness of this approach. Furthermore, errors or branch omissions during vessel extraction could cause misregistration of the data. The use of volume data in our approach has the added advantage of allowing MPS contour verification by anatomic CTA volume (Fig. 5), which would not be possible when only coronary tree data are coregistered. Others have previously proposed registration of MPS and unenhanced CT data obtained by

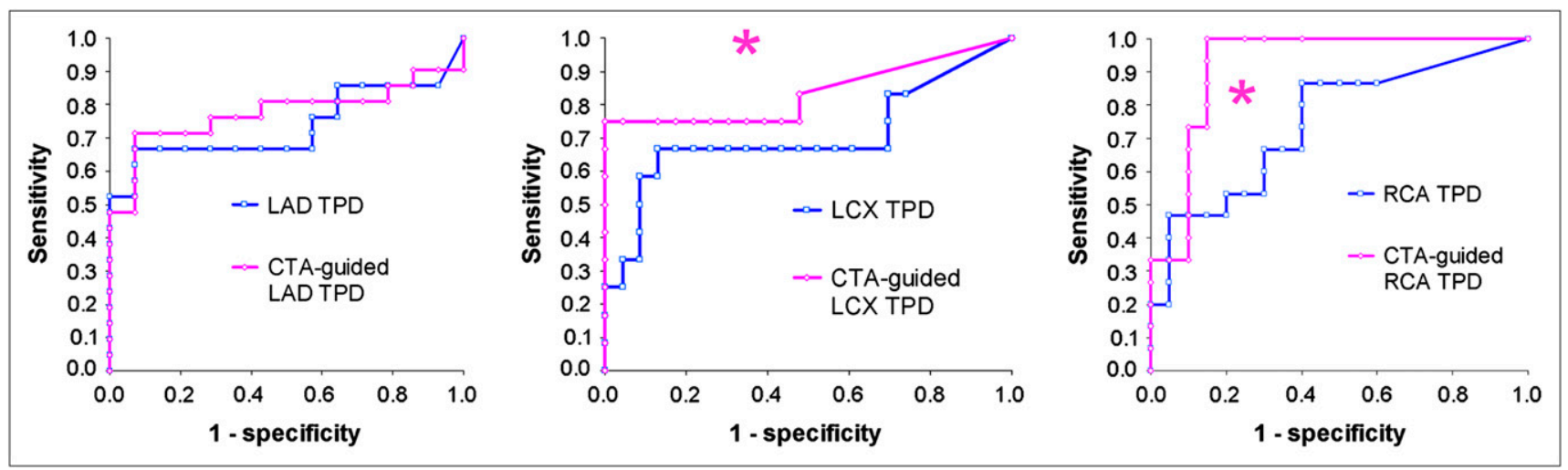

FIGURE 3. ROC curves for disease detection in individual vessels by partial TPD per vessel in stand-alone MPS (blue) and CTA-guided MPS (pink) for LAD (left), LCX (middle), and RCA (right) vascular territories. CTA-guided MPS significantly different from stand-alone MPS. 

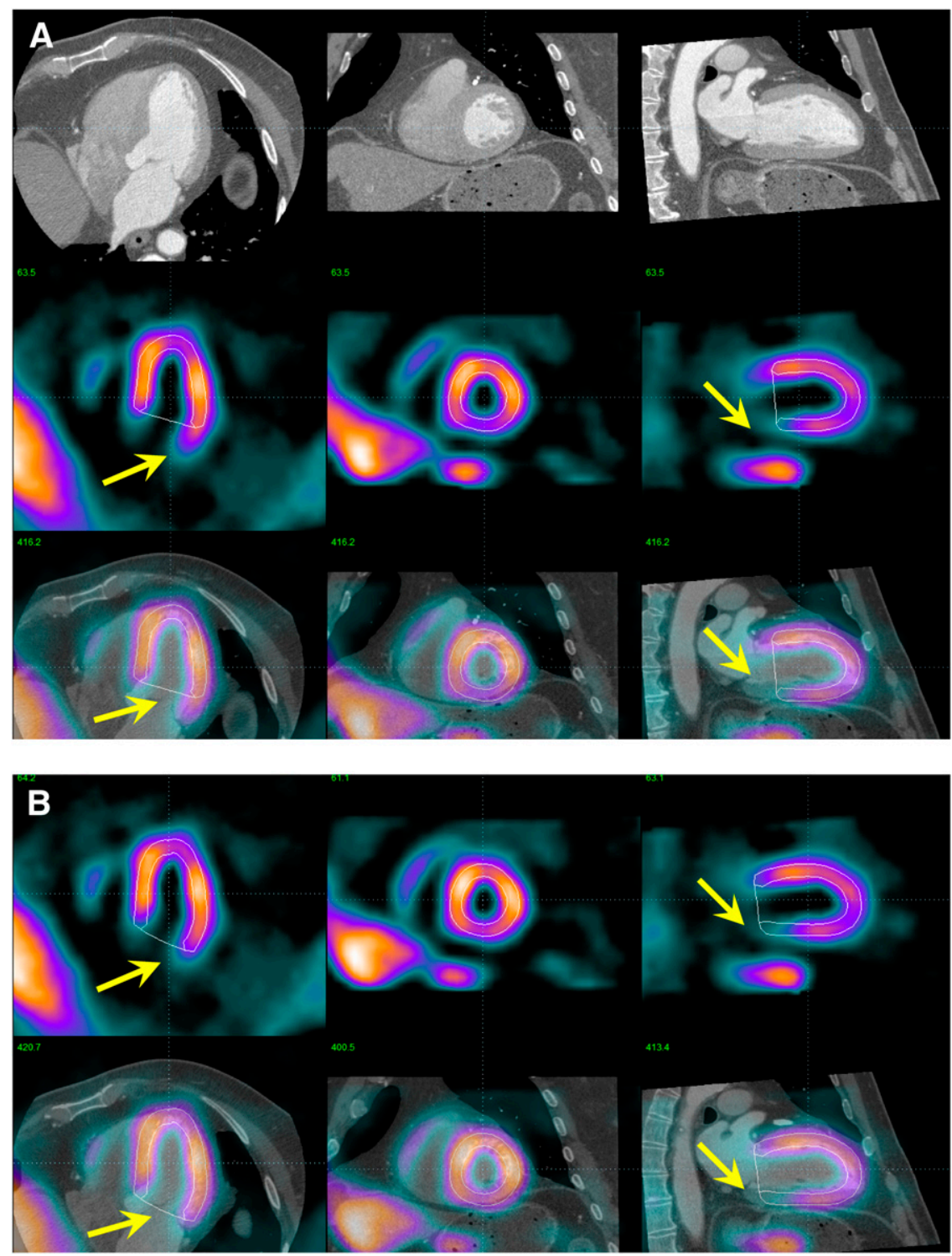

FIGURE 4. Example of MPS contour adjustment after MPS-CTA coregistration. (A) Coregistered CTA and MPS images are shown in same configuration as in Figure 2, with MPS contours overlaid (white lines). Image fusion reveals that valve plane is determined incorrectly (arrows). Same images are shown after MPS contour adjustment (B), revealing RCA defect on MPS. ICA confirmed $\geq 70 \%$ stenosis in RCA. hybrid scanners for the purposes of attenuation correction (26); however, in that application the data are already in an approximate alignment and only small correction is required. We have previously developed an MRI-MPS volume registration technique that used motion on MRI to presegment MRI heart for registration with MPS (27), but this approach could not be applied here because only 1 phase of the CTA data was available in this analysis. In addition, multiphase data are not available for prospectively gated CTA.

In this work, we used motion-frozen MPS data (11) for image registration with coronary CTA. A motion-frozen perfusion image corresponds to the ED phase of the cardiac cycle and is, therefore, better suited for fusion with coronary CTA, which is typically reconstructed in the $70 \%-80 \%$ phase for visualization of the coronary lesions. In previous MPS-CTA fusion studies from stand-alone or hybrid scanners $(4,23)$, summed MPS data were used for image fusion. This could potentially lead to mismatches in the apparent size of the ventricle. Motion-frozen MPS images have ED myocardial dimensions and wall thickness and therefore correspond more closely to CTA images.

The diagnostic results obtained for stand-alone CTA or MPS as shown in Figure 4 appear poor when compared with published reports on the respective performance of MPS and CTA modalities $(21,28)$. This is most likely due to the bias in our study population toward patients with frequent occurrences of equivocal results from the initial test and significant discrepancy between initial test interpretation and clinical suspicion. We demonstrated that, in such difficult cases, CTA-MPS image fusion and subsequent quantitative analysis can be helpful. Analysis of the diagnostic performance for specific vessels reveals that CTA-guided quantification was helpful in RCA and LCX territories but did not significantly improve the detection of LAD disease. This finding likely reflected the selective impact of basal contour adjustment on MPS. 

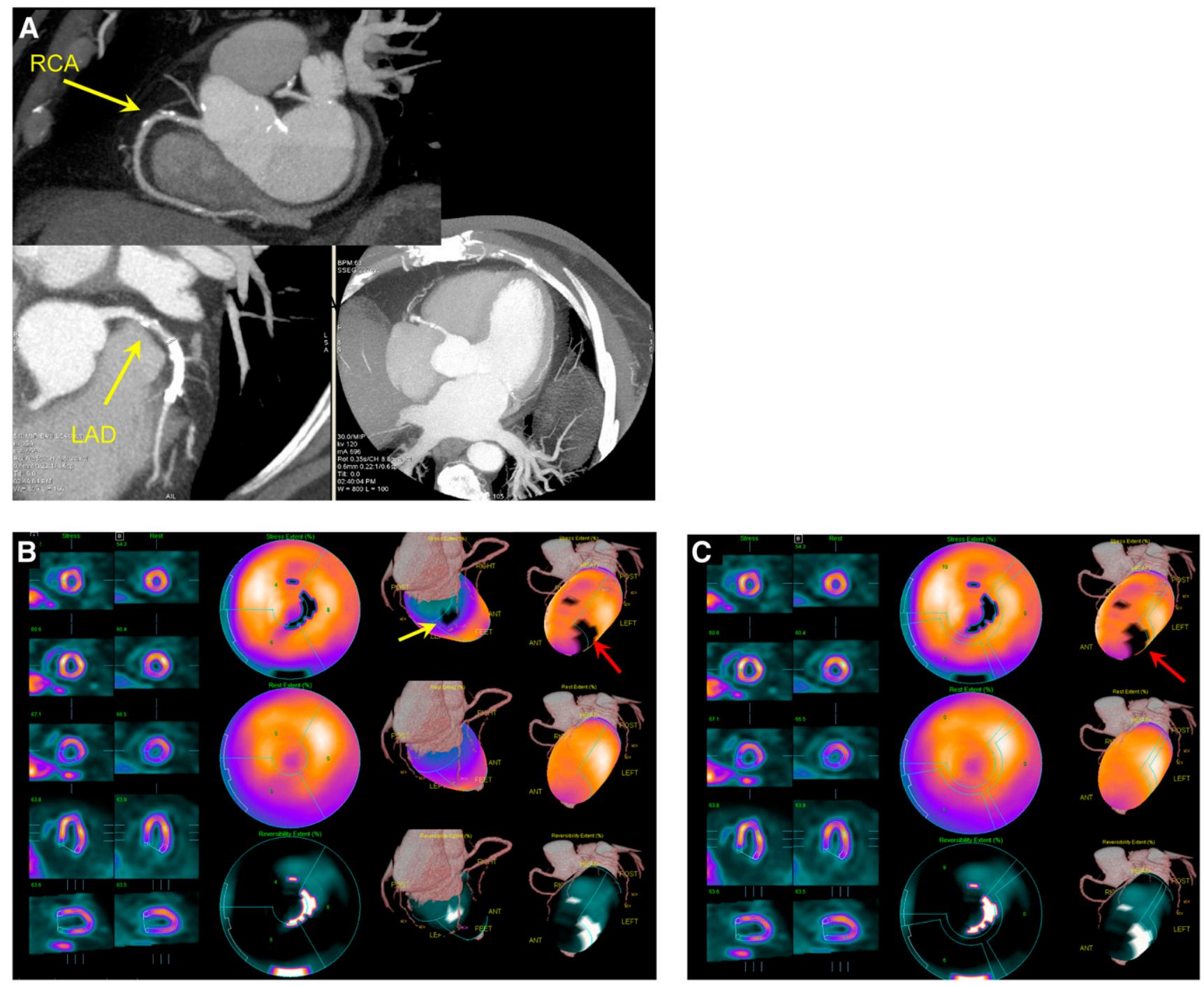

FIGURE 5. Example of MPS contour and territory adjustment based on CTA in 72-y-old male patient. Original coronary CTA images $(A)$ were interpreted to show nonsignificant, $<50 \%$ proximal RCA lesion and significant LAD lesion. CTA and MPS images were coregistered and indicated need for contour adjustment (as shown in Fig. 4). Quantification after contour adjustment reveals 3\% defect in typical RCA territory (yellow arrow) and defect between LAD and LCX (red arrow) (B). After adjustments of coronary territory on the basis of superimposed CTA coronary tree, MPS lesion is assigned to LAD (C). ICA revealed $50 \%-69 \%$ RCA lesion and $90 \%$ LAD lesion. In this case, CTA-guided analysis allowed identification of additional RCA lesion in MPS and reassignment of second defect from LAD/LCX to LAD.

This study analyzed the MPS-CTA data obtained by standalone scanners in 2 separate imaging sessions. Hybrid MPScoronary CTA (23) or PET-coronary CTA imaging (29) performed in 1 session has been proposed for PET/CT scanners equipped with multislice CT. However, although hybrid dual-modality imaging has been established in oncology (30), it is not used routinely in cardiac imaging because of the difficulty in predicting a priori which patients would benefit from such combined examination (31). Instead, a sequential approach is often applied in clinical practice, with additional scans (CTA or MPS) performed only if the results of the initial modality are equivocal —as was the situation in our retrospective analysis. In addition, even if MPS-CTA scans are obtained on a hybrid scanner, with data from both modalities in similar anatomic positions, software coregistration is still required because of mismatches in the respiratory phases (23). Because software registration can reliably bring MPS and CTA data acquired on different scanners into appropriate alignment as demonstrated in this work, the most practical approach for overall clinical effectiveness and the minimization of the cost and radiation dose may be the sequential scanning as presented here, facilitated by software tools for automatic image registration and combined image analysis.

Mean estimated radiation dose from the CT (CTA and coronary calcium scoring scan) was $19.7 \mathrm{mSv}$. For the dualisotope stress-rest MPS scans, the estimated radiation dose has been reported to be $24 \mathrm{mSv}$ for injected radiopharmaceutical activity similar to our protocol (32). The 2 scans 
were not obtained at the same time. Imaging teams at both sites have significantly reduced coronary CTA radiation dose by acquiring with prospective ECG gating when possible $(33,34)$ and using a patient-specific algorithm to select the optimal dose-lowering combination for retrospectively gated acquisitions, with a resulting mean estimated CTA radiation dose of $2-5.8 \mathrm{mSv}$ for prospective gating and $8 \mathrm{mSv}$ for retrospective gating (33-35). Additionally, we have changed our standard MPS protocol to ${ }^{99 m}$ Tc-sestamibi for both stress and rest (estimated radiation dose for this protocol, $10 \mathrm{mSv}$ ) (32). Thus, it is possible to perform a combined CTA and MPS study with the total dose less than $20 \mathrm{mSv}$, even with CTA retrospective gating. It should be emphasized that patients underwent either coronary CTA or stress-rest MPS first and only proceeded with dual scanning because of equivocal findings in the first scan or discrepancy between scan results and clinician assessment of patient status (typically in $<10 \%$ of the total patient population).

This study has several limitations. Although we provided fully automated quantitative analysis and automated image registration, the contour definitions and vascular territory were manually guided by the coregistered CTA anatomy; however, it is feasible that this adjustment can be automated in the future if automatic segmentation of CTA scan is performed. The success of registration depends on successful MPS contour determination. If the contours are incorrectly determined, causing the LV shape to be grossly distorted, the automatic registration could fail. We performed the analysis in a retrospective fashion, and therefore data selection was biased by clinical conditions that led to performance of invasive angiography, MPS, and CTA, which occurs in a minority of patients undergoing evaluation for CAD. However, these data likely represent typical cases in which the CTA-guided MPS quantification could be clinically useful.

\section{CONCLUSION}

Software coregistration of coronary CTA and MPS images obtained on separate scanners can be acquired rapidly and automatically, allowing CTA-guided contour and vascular territory adjustment on MPS for improved quantitative MPS analysis.

\section{ACKNOWLEDGMENTS}

Cedars-Sinai Medical Center receives or may receive royalties for the licensure of software, including software for quantitative assessment of myocardial perfusion and for MPS CT fusion, a portion of which is distributed to some of the authors of this manuscript.

\section{REFERENCES}

1. Leber AW, Becker A, Knez A, et al. Accuracy of 64-slice computed tomography to classify and quantify plaque volumes in the proximal coronary system: a comparative study using intravascular ultrasound. J Am Coll Cardiol. 2006;47: 672-677.
2. Berman DS, Hachamovitch R, Shaw LJ, et al. Roles of nuclear cardiology, cardiac computed tomography, and cardiac magnetic resonance: assessment of patients with suspected coronary artery disease. J Nucl Med. 2006;47:74-82.

3. Hendel R, Patel M, Kramer C, et al. Appropriateness criteria for cardiac computed tomography and cardiac magnetic resonance imaging. J Am Coll Cardiol. 2006;48:1475-1497.

4. Gaemperli O, Schepis T, Valenta I, et al. Cardiac image fusion from stand-alone SPECT and CT: clinical experience. J Nucl Med. 2007;48:696-703.

5. Gaemperli O, Schepis T, Kalff V, et al. Validation of a new cardiac image fusion software for three-dimensional integration of myocardial perfusion SPECT and stand-alone 64-slice CT angiography. Eur J Nucl Med Mol Imaging. 2007;34: 1097-1106.

6. Agatston AS, Janowitz WR, Hildner FJ, Zusmer NR, Viamonte M, Detrano R. Quantification of coronary artery calcium using ultrafast computed tomography. J Am Coll Cardiol. 1990;15:827-832.

7. Berman DS, Wong ND, Gransar H, et al. Relationship between stress-induced myocardial ischemia and atherosclerosis measured by coronary calcium tomography. J Am Coll Cardiol. 2004;44:923-930.

8. Ferencik M, Ropers D, Abbara S, et al. Diagnostic accuracy of image postprocessing methods for the detection of coronary artery stenoses by using multidetector CT. Radiology. 2007;243:696-702.

9. Berman DS, Kiat H, Friedman JD, et al. Separate acquisition rest thallium-201/ stress technetium-99m sestamibi dual-isotope myocardial perfusion singlephoton emission computed tomography: a clinical validation study. J Am Coll Cardiol. 1993;22:1455-1464.

10. Berman DS, Kang X, Hayes SW, et al. Adenosine myocardial perfusion singlephoton emission computed tomography in women compared with men: impact of diabetes mellitus on incremental prognostic value and effect on patient management. J Am Coll Cardiol. 2003;41:1125-1133.

11. Slomka PJ, Nishina H, Berman DS, et al. "Motion-frozen" display and quantification of myocardial perfusion. J Nucl Med. 2004;45:1128-1134.

12. Germano G, Kavanagh PB, Berman DS. An automatic approach to the analysis, quantitation and review of perfusion and function from myocardial perfusion SPECT images. Int J Card Imaging. 1997;13:337-346.

13. Germano G, Erel J, Lewin H, Kavanagh PB, Berman DS. Automatic quantitation of regional myocardial wall motion and thickening from gated technetium-99m sestamibi myocardial perfusion single-photon emission computed tomography. J Am Coll Cardiol. 1997;30:1360-1367.

14. Germano G, Kiat H, Kavanagh PB, et al. Automatic quantification of ejection fraction from gated myocardial perfusion SPECT. J Nucl Med. 1995;36: 2138-2147.

15. Bookstein FL. Principal warps: thin-plate splines and the decomposition of deformations. IEEE Trans Pattern Anal Machine Intell. 1989;11:567-585.

16. Slomka PJ, Dey D, Przetak C, Aladl UE, Baum RP. Automated 3-dimensional registration of stand-alone ${ }^{18}$ F-FDG whole-body PET with CT. J Nucl Med. 2003;44:1156-1167.

17. Viola P, Wells WM. Alignment by maximization of mutual information. Int $J$ Comput Vis. 1997;24:137-154.

18. Ibáñez L, Schroeder W, Ng L, Cates J. The ITK Software Guide. Kitware; 2003. Available at: http://www.itk.org/ItkSoftwareGuide.pdf. Accessed July 28, 2009.

19. Levin D, Aladl UE, Germano G, Slomka P. Techniques for efficient, real-time, 3D visualization of multimodality cardiac data using consumer graphics hardware. Comput Med Imaging Graph. 2004;28:471-483.

20. Sharir T, Germano G, Waechter PB, et al. A new algorithm for the quantitation of myocardial perfusion SPECT. II: validation and diagnostic yield. J Nucl Med. 2000;41:720-727.

21. Slomka PJ, Nishina H, Berman DS, et al. Automated quantification of myocardial perfusion SPECT using simplified normal limits. J Nucl Cardiol. 2005;12:66-77.

22. DeLong ER, DeLong DM, Clarke-Pearson DL. Comparing the areas under two or more correlated receiver operating characteristic curves: a nonparametric approach. Biometrics. 1988;44:837-845.

23. Rispler S, Keidar Z, Ghersin E, et al. Integrated single-photon emission computed tomography and computed tomography coronary angiography for the assessment of hemodynamically significant coronary artery lesions. J Am Coll Cardiol. 2007;49:1059-1067.

24. Faber TL, Santana CA, Garcia EV, et al. Three-dimensional fusion of coronary arteries with myocardial perfusion distributions: clinical validation. $J$ Nucl Med. 2004;45:745-753.

25. Besl P, McKay N. A method for registration of 3D shapes. IEEE Trans Pattern Anal Mach Intell. 1992;14:239-256.

26. Guetter C, Wacker M, Xu C, Hornegger J. Registration of cardiac SPECT/CT data through weighted intensity co-occurrence priors. Lect Notes Comput Sci. 2007;4791:725-733. 
27. Aladl UE, Hurwitz GA, Dey D, Levin D, Drangova M, Slomka PJ. Automated image registration of gated cardiac single-photon emission computed tomography and magnetic resonance imaging. J Magn Reson Imaging. 2004;19: 283-290.

28. Delago A, Min JK, Halamert E, et al. Diagnostic performance of 64multidetector row coronary computed tomographic angiography for evaluation of coronary artery stenosis in individuals without known coronary artery disease: results from the prospective multicenter ACCURACY (Assessment by Coronary Computed Tomographic Angiography of Individuals Undergoing Invasive Coronary Angiography) trial. J Am Coll Cardiol. 2008;52:1733-1735.

29. Di Carli MF, Dorbala S, Hachamovitch R. Integrated cardiac PET-CT for the diagnosis and management of CAD. J Nucl Cardiol. 2006;13:139144.

30. Townsend DW. Dual-modality imaging: combining anatomy and function. J Nucl Med. 2008;49:938-955.
31. Slomka PJ, Berman DS, Germano G. Applications and software techniques for integrated cardiac multimodality imaging. Expert Rev Cardiovasc Ther. 2008;6: 27-41.

32. Einstein AJ. Radiation risk from coronary artery disease imaging: how do different diagnostic tests compare? Heart. 2008;94:1519-1521.

33. Gutstein A, Wolak A, Lee CJ, et al. Predicting success of prospective and retrospective gating with dual-source coronary computed tomography angiography: development of selection criteria and initial experience. J Cardiovasc Comput Tomogr. 2008;2:81-90.

34. Gopal A, Mao SS, Karlsberg D, et al. Radiation reduction with prospective ECGtriggering acquisition using 64-multidetector computed tomographic angiography. Int J Cardiovasc Imaging. 2009;25:405-416.

35. Gutstein A, Dey D, Cheng V, et al. Algorithm for radiation dose reduction with helical dual source coronary computed tomography in clinical practice. J Cardiovasc Comput Tomogr. 2008;2:311-322. 